\title{
Estilos de liderança situacional adotados pelos enfermeiros na área hospitalar
}

Karen Cristina Kades Andrigue ${ }^{1}$, Leticia de Lima Trindade ${ }^{2}$, Simone Coelho Amestoy ${ }^{3}$, Carmem Lúcia Colomé Beck ${ }^{4}$

\footnotetext{
${ }^{1}$ Enfermeira, Mestre em Ciências da Saúde. Docente da Universidade Comunitária da Região de Chapecó. Chapecó, SC, Brasil. E-mail: karenandrigue@unochapeco.edu.br.

${ }^{2}$ Enfermeira, Doutora em Enfermagem. Professor Adjunto da Universidade do Estado de Santa Catarina. Chapecó, SC, Brasil. E-mail:

leticia.trindade@unochapeco.edu.br.

${ }^{3}$ Enfermeira, Doutora em Enfermagem. Professor Adjunto da Universidade Federal de Pelotas. Pelotas, RS, Brasil. E-mail: simoneamestoy@hotmail.com.

${ }^{4}$ Enfermeira, Doutora em Enfermagem. Professor Associado da Universidade Federal de Santa Maria. Santa Maria, RS, Brasil. E-mail: carmembeck@gmail.com.
}

Recebido: 04/04/2016

Aceito: 02/09/2016.

Publicado: 12/12/2016

Como citar esse artigo:

Andrigue KCK, Trindade LL, Amestoy SC, Beck CLC. Estilos de liderança situacional adotados pelos enfermeiros na área hospitalar. Rev. Eletr. Enf. [Internet]. 2016 [acesso em:_____ ] 18:e1191. Disponível em: http://dx.doi.org/10.5216/ree.v18.40551.

\section{RESUMO}

Objetivou-se analisar estilos de Liderança Situacional adotados por enfermeiros hospitalares e a associação destes com seu perfil pessoal e profissional. Estudo quantitativo, descritivo, realizado com enfermeiros que atuam no contexto hospitalar e que utilizou o modelo de liderança de Hersey e Blanchard. Os dados foram coletados por meio de questionário sociodemográfico e pelo Leadership Effectiveness and Adaptability Description. $\mathrm{O}$ estilo de liderança mais diretivo, focado na capacidade de persuasão foi o mais presente. 0 setor de atuação apresentou relação significativa ao estilo de liderança $(p=00,1)$, demonstrando aproximação entre assistência e perfil de liderança. Embora a população integre um grupo jovem, com pouco tempo de atuação, o predomínio do estilo de liderança Persuadir pode limitar a criatividade e potencialidade dos integrantes da equipe pelo foco ser centrado na tarefa. Estratégias que permitam alcançar elevados níveis de maturidade, podem auxiliar enfermeiros na adoção de práticas de liderança mais flexíveis.

Descritores: Pesquisa em Administração de Enfermagem; Liderança; Prática Profissional; Serviços de Saúde.

\section{INTRODUÇÃO}

Para a Enfermagem, a liderança emerge como o processo por meio do qual o enfermeiro influencia as ações de outros para a determinação e a consecução de objetivos, buscando promover mudança na sua prática diária, com vistas à melhoria da qualidade da assistência prestada ao usuário ${ }^{(1)}$. No contexto da maioria dos serviços de saúde no Brasil, o enfermeiro é reconhecido como líder por exercer a posição de coordenador da equipe de enfermagem e de gerente de unidades e instituições hospitalares, sendo 
reconhecido como profissional de referência ${ }^{(2)}$.

Estudos internacionais apontam para a necessidade de fortalecer a liderança na enfermagem, por intermédio de programas de desenvolvimento de líderes. A liderança tem sido associada à melhora da cultura organizacional, satisfação pessoal, melhor interação interpessoal e confiança entre os líderes e a equipe ${ }^{(3-4)}$, e, mais importante, melhorou os resultados relacionados ao atendimento aos pacientes ${ }^{(5)}$.

Quando exercida de forma positiva, eleva a qualidade da assistência prestada ao usuário, pois esta é influenciada de forma significativa pelo líder e pela maneira como este gerencia o serviço em termos ambientais, humanos ou técnicos ${ }^{(6)}$ e consideram ainda que a satisfação e confiança na execução dos processos de trabalho diários são diretamente influenciados pelas relações de liderança ${ }^{(7)}$. $O$ estilo de liderança é o padrão comportamental que uma pessoa manifesta quando procura influenciar as atividades de outras ${ }^{(8)}$. Nesse sentido, a Liderança Situacional emerge como um conceito integrador, que objetiva que o sujeito se aproprie de seu processo de liderança ${ }^{(8)}$.

Para este estudo utilizou-se o referencial teórico de Hersey e Blanchard, denominado Liderança Situacional (LS), que se baseia em quatro estilos de liderar, sendo eles Determinar, Persuadir, Compartilhar e Delegar, os quais consistem em uma combinação de comportamento de tarefa e de relacionamento. 0 comportamento de tarefa representa a medida com que o líder dirige as pessoas, dizendo-lhes o que, quando, onde e como fazer determinada tarefa. Já o comportamento direcionado ao relacionamento considera a capacidade do líder em comunicar-se, ouvir e apoiar seus colaboradores ${ }^{(7)}$.

Pesquisas ${ }^{(6,9-11)}$ em diferentes países têm considerado a influência dos estilos de liderança como estratégia para qualificar o cuidado ao usuário e sua família quando esta possui alta qualidade, é permeada pela confiança, boa comunicação, respeito e reciprocidade ${ }^{(3)}$. No cenário nacional, a LS vem sendo estudada e apontada como estratégia de qualificação aos processos de liderar ${ }^{(1,3)}$, visto que sua aplicação constitui um dos caminhos para viabilizar o aprendizado sobre liderança e a formação de enfermeiros-líderes ${ }^{(1)}$.

Nessa direção, questionou-se: quais estilos de LS são adotados pelos enfermeiros na área hospitalar? De que forma o perfil pessoal e profissional, bem como a formação profissional (acadêmica e EPS), influenciam a liderança do enfermeiro?

Nesse sentido, este estudo objetivou analisar os estilos de Liderança Situacional adotados pelos enfermeiros de uma rede hospitalar, bem como a associação destes com o perfil pessoal e profissional dos participantes.

\section{MÉTODO}

Estudo quantitativo, realizado em três hospitais que compõem a rede hospitalar de alta complexidade da região Sul do Brasil. Os cenários selecionados se destacam como polo de referência em diversas áreas de complexidade. Em conjunto, os hospitais totalizam mais de 500 leitos de internação.

Conforme consultoria estatística, após a aplicação do teste de amostragem para este numérico, a amostra deveria ser composta por 87 participantes. Mediante interesse de aumentar o intervalo de 
confiança, ampliou-se a amostra para 104 enfermeiros, garantindo uma margem de erro menor a 5\% e intervalo de confiança de 95 .

Como critérios de inclusão, optou-se: possuir vínculo profissional como enfermeiro nos cenários e atuar nos diferentes turnos de trabalho. Foram excluídos do estudo somente oito profissionais afastados por adoecimento e os contratados como trainee, pois estes atuam sob supervisão de um enfermeiro, caracterizando o processo de trabalho como treinamento. Os enfermeiros participantes exerciam suas atividades em setores assistenciais e gerenciais, sendo eles: Unidades de Internação (UI); Centro Cirúrgico (CC); Central de Materiais Esterilizados (CME). Ainda participaram enfermeiros das Unidades Especiais (UE), para as quais se agrupou o Serviço de Controle de Infecções Relacionadas à Assistência à Saúde (SCIRAS); Serviço de Educação Permanente em Enfermagem (SEPE); Núcleo Hospitalar de Epidemiologia (NHE); Central de Captação e Transplante de Órgãos e Tecidos (CIDOTH); e Unidades Ambulatoriais (UA), que incluíram: Urgência e Emergência, Quimioterapia, Radioterapia, Ortopedia e Traumatologia e Serviço Diagnóstico. Para coleta de dados, solicitou-se o preenchimento pelos participantes do Questionário Sociodemográfico para caracterização do perfil e do Leadership Effectiveness and Adaptability Description (LEAD) - Descrição da Eficácia e de Adaptabilidade do Líder, o qual foi desenvolvido no Center for Leadership Studies da Universidade de Ohio $^{(12)}$. A utilização do instrumento foi fundamentada na adaptação realizada para a área da enfermagem ${ }^{(13)}$.

O LEAD é composto de 12 "situações" com quatro alternativas de ação, devendo o participante optar por uma das ações, a qual mais se adeque a seu comportamento de liderança nas situações apresentadas. A categorização quanto aos estilos de liderança seguiu o instrumento de conversão $L E A D$.

$O$ instrumento categoriza os estilos de liderança em: Determinar (E1), na adoção deste perfil o enfermeiro define a atividade, como, quando e onde deve ser executada e supervisiona constantemente a execução da tarefa; Persuadir (E2), o enfermeiro explica a atividade a ser executada, ouve o colaborador atentamente e busca convencê-lo da forma mais apropriada para realizar a tarefa; Compartilhar (E3), o enfermeiro possibilita que o colaborador participe da tomada de decisão, ou seja, compartilha as ideias e as alternativas para resolver determinado problema; e Delegar (E4), para o qual frente a uma tarefa específica o colaborador decide como, quando e onde fazer as coisas, implica para o enfermeiro comportamento baixo tanto para tarefa como para relacionamento ${ }^{(1,7-8)}$.

Os dados foram coletados no período de junho a agosto de 2014 in loco, mediante autorização das instituições e agendamento prévio. Para a análise e organização dos achados, estes foram duplamente digitados e tabulados no Programa Microsoft Excel 2010 (Microsoft Corp.) e submetidos ao tratamento estatístico utilizando-se o programa Statistical Package for the Social Sciences (SPSS), versão 21. As variáveis categóricas foram apresentadas em frequência absoluta e relativa e as numéricas em média e desvio-padrão. Utilizou-se o teste qui-quadrado para comparação de grupos de participantes por instituições quanto as variáveis categóricas. O nível de significância adotado foi de 0,05 (5\%) para diferenças significantes.

O estudo foi conduzido atendendo as normas brasileiras para pesquisas com seres humanos. 
Conforme determinado, foi garantido aos participantes o anonimato, o sigilo, o direito de desistir do estudo, o livre acesso aos dados quando de seu interesse, mediante assinatura por ambas as partes do Termo de Consentimento Livre e Esclarecido (em duas vias). O estudo foi submetido e aprovado pelo Comitê de Ética em Pesquisa da Santa Casa de Misericórdia de Pelotas (aprovação sob n. 200/2013). Os achados serão guardados pelos pesquisadores por cinco anos.

\section{RESULTADOS}

Participaram do estudo enfermeiros que atuam em três hospitais, estes contam em conjunto com 112 enfermeiros contratos e registrados, contudo, participaram 104 profissionais (92,85\%), sendo que 65,18\% da amostra atuava no hospital I e $12,50 \%$ no hospital III, nos quais se alcançou todos os enfermeiros contratados ( $n=73 / n=14$, respectivamente). O hospital II possuía, no momento da coleta de dados, 25 enfermeiros, destes, 17 participaram do estudo (22,32\% da amostra).

Para melhor ilustrar os participantes do estudo, utilizou-se a Tabela 1.

Tabela 1: Demonstrativo dos enfermeiros, segundo instituições selecionadas e participação no estudo. Chapecó, SC, Brasil, 2015.

\begin{tabular}{ccccc}
\hline Hospital & Total de profissionais & \% & Participantes do estudo & \% \\
\hline H - I & 73 & 65.18 & 73 & 100 \\
H - II & 25 & 22.32 & 17 & 68 \\
H - III & 14 & 12.50 & 14 & 100 \\
\hline Total & $\mathbf{1 1 2}$ & $\mathbf{1 0 0}$ & $\mathbf{1 0 4}$ & $\mathbf{9 2 . 8 5}$ \\
\hline
\end{tabular}

Dentre os participantes houve o predomínio de mulheres ( $n=90-86,5 \%$ ), com idade média de $32,1 \pm 6,5$ anos, na faixa etária entre $21 \vdash 30$ anos $(n=48-46,2 \%)$, as quais na maioria atuam na área hospitalar entre $1 \vdash 5$ anos $(n=38-36,5 \%)$.

Quanto ao perfil de formação, o tempo de graduação predominou de $1 \vdash 5$ ( $n=46-44,2 \%$ ) e parte representativa da amostra ( $n=86-82,7 \%$ ) possui curso de especialização, enquanto que somente $3,8 \%$ dos profissionais cursaram mestrado $(n=4)$.

Referente ao setor de atuação, 63,5\% (n=66) trabalham em Unidades de Internação e 73,1\% (n=76) ocupam cargos como enfermeiros assistenciais, dentre os quais $29,8 \%(n=31)$ atuam no turno matutino. Observou-se que $76,9 \%(n=80)$ não possuíam outros vínculos de trabalho e um mesmo percentual atua 44 horas semanais. Cabe destacar que todos os enfermeiros participantes do estudo possuem contrato de trabalho regido pela Consolidação das Leis de Trabalho (CLT).

A Tabela 2 permite a identificação da distribuição de todos os participantes por características sociodemográficas e profissionais.

Ao analisar o estilo de LS adotado, o estudo evidenciou o predomínio do estilo E2 (Persuadir), com o qual $68,2 \%$ dos participantes ( $n=71$ ) se autoidentificaram. Na sequência, destacaram-se os participantes autorreferidos no E1 (Determinar) ( $n=18-17,3 \%)$ e os participantes com E3 (Compartilhar) ( $n=10-9,6 \%)$. Os demais participantes analisaram-se em mais de um estilo e nenhum participante avaliou-se no E4 (Delegar). 
Tabela 2: Perfil dos enfermeiros da rede hospitalar. Chapecó, SC, Brasil, 2015.

\begin{tabular}{|c|c|c|c|c|c|c|c|c|c|}
\hline \multirow{2}{*}{ Variáveis } & \multicolumn{2}{|c|}{$H-I(n=73)$} & \multicolumn{2}{|c|}{$H-I I(n=14)$} & \multicolumn{2}{|c|}{$H-I I I(n=17)$} & \multicolumn{2}{|c|}{ Total $(n=104)$} & \multirow{2}{*}{ Valor $\mathrm{p}^{*}$} \\
\hline & $\mathbf{n}$ & $\%$ & $\mathbf{n}$ & $\%$ & $\mathbf{n}$ & $\%$ & n & $\%$ & \\
\hline Sexo & & & & & & & & & 0,662 \\
\hline Feminino & 61 & 83,6 & 14 & 100 & 15 & 88,2 & 90 & 86,5 & \\
\hline Masculino & 12 & 16,4 & 0 & 0 & 2 & 11,8 & 14 & 13,5 & \\
\hline Faixa Etária & & & & & & & & & 0,863 \\
\hline $21-30$ & 33 & 45,2 & 6 & 42,9 & 9 & 52,9 & 48 & 46,2 & \\
\hline $31-40$ & 30 & 41,1 & 7 & 50,5 & 7 & 41,2 & 44 & 42,3 & \\
\hline $41-50$ & 9 & 12,3 & 1 & 7,1 & 1 & 5,9 & 11 & 10,6 & \\
\hline $51-60$ & 1 & 1,4 & 0 & 0 & 0 & 0 & 1 & 1 & \\
\hline Tempo de trabalho & & & & & & & & & 0,310 \\
\hline$<1$ ano & 15 & 20,5 & 5 & 35,7 & 5 & 29,4 & 25 & 24 & \\
\hline 01/mai & 27 & 37 & 6 & 42,9 & 5 & 29,4 & 38 & 36,5 & \\
\hline 05/out & 16 & 21,9 & 2 & 14,3 & 5 & 29,4 & 23 & 22,1 & \\
\hline$>10$ & 15 & 20,5 & 1 & 7,1 & 2 & 11,8 & 18 & 17,3 & \\
\hline Setor de atuação & & & & & & & & & 0,001 \\
\hline UI & 45 & 61,6 & 8 & 57,1 & 13 & 76,5 & 66 & 63,5 & \\
\hline CC e CME & 9 & 12,3 & 3 & 21,4 & 0 & 0 & 12 & 11,5 & \\
\hline UE & 7 & 9,6 & 1 & 7,1 & 0 & 0 & 8 & 7,7 & \\
\hline UA & 12 & 16,4 & 2 & 14,3 & 4 & 23,5 & 18 & 17,3 & \\
\hline Tipo de cargo de chefia & & & & & & & & & 0,217 \\
\hline Gerência & 1 & 1,4 & 1 & 7,1 & 0 & 0 & 2 & 1,9 & \\
\hline Coordenação & 24 & 32,9 & 0 & 0 & 2 & 11,8 & 26 & 25 & \\
\hline Assistência & 48 & 65,8 & 13 & 92,99 & 15 & 88,2 & 76 & 73,1 & \\
\hline Turno de trabalho & & & & & & & & & 0,412 \\
\hline Matutino & 17 & 23,3 & 6 & 42,9 & 8 & 47,1 & 31 & 29,8 & \\
\hline Vespertino & 19 & 26 & 4 & 28,6 & 3 & 17,6 & 26 & 25 & \\
\hline Noturno & 20 & 27 & 3 & 21,4 & 5 & 29,4 & 28 & 26,9 & \\
\hline $\mathrm{HC}$ & 17 & 23,3 & 1 & 7,1 & 1 & 5,9 & 19 & 18,3 & \\
\hline Outros vínculos & & & & & & & & & 0,305 \\
\hline Sim & 16 & 21,9 & 4 & 28,6 & 4 & 23,5 & 24 & 23,1 & \\
\hline Não & 57 & 78,1 & 10 & 71,4 & 13 & 76,5 & 80 & 76,9 & \\
\hline Horas semanais trabalhadas & & & & & & & & & 0,497 \\
\hline$\leq 44$ & 57 & 78,1 & 10 & 71,4 & 13 & 76,5 & 80 & 76,9 & \\
\hline $45-65$ & 10 & 13,7 & 2 & 14,3 & 1 & 5,9 & 13 & 12,5 & \\
\hline $66-85$ & 4 & 5,5 & 2 & 14,3 & 1 & 5,9 & 7 & 6,7 & \\
\hline$>85$ & 2 & 2,7 & 0 & 0 & 2 & 11,8 & 4 & 3,8 & \\
\hline
\end{tabular}

A Tabela 3 permite compreender a distribuição dos enfermeiros por estilo de liderança adotado, observando que: no H-1, o E2 (Persuadir) abrangeu 58,9\% dos participantes ( $n=43$ ); no H-2, 88,2\% ( $n=15$ ); e no $H-3,92,9 \%(n=13)$.

Tabela 3: Estilos de Liderança Situacional. Chapecó, SC, Brasil, 2015.

\begin{tabular}{|c|c|c|c|c|c|c|c|c|c|}
\hline \multirow{2}{*}{ Variáveis } & \multicolumn{2}{|c|}{ E1 - Determinar $(n=18)$} & \multicolumn{2}{|c|}{ E2 - Persuadir ( $n=71)$} & \multicolumn{2}{|c|}{ E3 - Compartilhar $(n=10)$} & \multicolumn{2}{|c|}{ E.* - Secundários $(n=5)$} & \multirow{2}{*}{ Valor $p$} \\
\hline & $n$ & $\%$ & $\mathbf{N}$ & $\%$ & $\mathbf{N}$ & $\%$ & $n$ & $\%$ & \\
\hline UI & 11 & 16,7 & 46 & 69,7 & 7 & 10,6 & 2 & 3 & 0,001 \\
\hline $\mathrm{CC} / \mathrm{CME}$ & 3 & 25 & 8 & 66,7 & 1 & 8,3 & 0 & 0 & \\
\hline UE & 4 & 50 & 2 & 25 & 0 & 0 & 2 & 25 & \\
\hline UA & 0 & 0 & 15 & 83,3 & 2 & 11,1 & 1 & 5,6 & \\
\hline Total & 18 & 17,3 & 71 & 68,3 & 10 & 9,6 & 5 & 4,8 & \\
\hline
\end{tabular}


Retornando à Tabela 1, observou-se que o setor de atuação possui relação significativa com relação ao estilo de liderança adotado pelos enfermeiros $(p=00,1)$. 0 estilo de liderança E2 (Persuadir) obteve maior prevalência entre os enfermeiros dos setores $\mathrm{UA}, \mathrm{CME} / \mathrm{CC}$ e UI, com 68,3\% ( $n=71$ ). O E1 (Determinar) prevaleceu nas UE, autorreferido por 22,2\% ( $n=18)$ dos enfermeiros. Já o E3 (Compartilhar) incidirem 9,6\% ( $n=10)$ da amostra e teve sua maior ocorrência nas UA ( $n=2-11,1 \%)$.

\section{DISCUSSÃO}

Observou-se o predomínio do sexo feminino entre os participantes, aspecto convergente com as pesquisas nacionais ${ }^{(14-15)} \mathrm{e}$ internacionais ${ }^{(3,9,11,16)}$. A faixa etária identificada foi ao encontro das últimas informações divulgadas pelo Conselho Federal de Enfermagem, o qual aponta que a equipe de Enfermagem é considerada jovem com $63,9 \%$ dos profissionais com idade igual ou inferior a 40 anos $^{(14)}$.

Ao contrário do que apresenta a Teoria de LS, a qual descreve ser mais fácil aos jovens alcançarem os estilos E3 (Compartilhar) e E4 (Delegar) ${ }^{(8)}$, os enfermeiros autoanalisaram-se mais no estilo E2 (Persuadir). Contudo, no estudo não se obteve relação significativa entre os estilos de liderança e a idade dos participantes $(p>0,05)$.

A ausência de enfermeiros no estilo delegar indicia para a dificuldade de compartilhar tarefas com a equipe e pode evidenciar a fragilidade dos profissionais diante do gerenciamento de pessoas e sua percepção quanto ao comprometimento organizacional. A adoção do estilo delegar poderia contribuir para a união e envolvimento da equipe em torno de um objetivo, refletindo como um fator potencializador de oportunidades para o fortalecimento da instituição e dos profissionais.

A adoção predominante do E2 (Persuadir) também foi encontrada em outros estudos no cenário hospitalar ${ }^{(1,9)}$, sugerindo um padrão de liderança que sofre influência da complexidade do trabalho nesses locais e da necessidade da tomada de decisões rápidas pelo enfermeiro com baixa interação com os demais membros da equipe de trabalho. Ressalta-se que a adoção de estilos diretivos pode influenciar negativamente as expectativas dos colaboradores em relação ao líder ${ }^{(16-17)}$ e ocasionar a erosão da confiança entre estes ${ }^{(18)}$. O estilo adotado influencia a satisfação no trabalho à medida que cada estilo é exercido ou não de acordo com o contexto situacional do trabalho da enfermagem, e dos múltiplos aspectos que o rodeiam, bem como da teia das relações e condutas.

Compreende-se, de modo geral, que os auxiliares e técnicos de enfermagem esperam que a atuação do líder ocorra de forma conjunta com a equipe, no desenvolvimento das atividades, na construção de rotinas e na tomada de decisão, favorecendo a satisfação no processo de trabalho ${ }^{(2,3,18)}$, aspectos que, por vezes, contrapõem-se diretamente ao estilo de persuasão e aproxima-se dos estilos Compartilhar ou Delegar.

Ainda, ressalta-se que a pequena parte dos participantes se identificou no estilo E3 (Compartilhar), sendo este mais autorreferido pelos enfermeiros mais jovens mais ao encontro da Teoria de LS. Estudiosos ${ }^{(19)}$ chamam a atenção para o impacto significativo de líderes de enfermagem na linha de frente na prestação de cuidados de alta qualidade, o que inclui a sua influência fundamental sobre a sustentabilidade a longo prazo 
das melhorias das práticas de enfermagem. Neste sentido, os líderes são encorajados atuar visando atender as prioridades da organização, bem como ter expectativas claras em relação a equipe de enfermagem.

O predomínio dos estilos E1 (Determinar) e E2 (Persuadir) indica a importância da educação permanente entre as equipes, integralizando-os com maior e menor tempo de formação, bem como profissionais com diferentes idades, na busca do fortalecimento da liderança. Para garantir o desenvolvimento de habilidades necessárias para exercer a liderança, os enfermeiros precisam ter acesso fácil à educação por meio de programas educacionais contemporâneos e de alta qualidade, que fortaleça a clínica e as competências gerenciais ${ }^{(20)}$.

Quanto ao duplo vínculo de emprego, este foi referido por uma parcela pequena da amostra $(23,1 \%)$, aspecto incomum entre os enfermeiros brasileiros ${ }^{(15)}$. Em parte, esse achado por ser justificado pela elevada carga horária semanal de trabalho dos participantes. Sabendo-se que o trabalho da enfermagem requer o convívio com os usuários em situação de dor, sofrimento e doença, turnos ininterruptos, em sábados, domingos e feriados, más condições de trabalho, muita responsabilidade e pouca valorização ${ }^{(15)}$.

Relacionado à formação, convém destacar que 81,7\% graduaram-se após instituídas as Diretrizes Curriculares Nacionais (DCN) para os cursos de Graduação em Enfermagem, no ano de 2001, as quais estão baseadas em competências gerenciais, entre elas liderança. A maioria possuía especialização e somente 3,8\% possuíam mestrado, dado comum pelo expressivo número de profissionais que apresentam somente especialização, e pelo maior contingente de enfermeiros mestres e doutores atuarem em hospitais universitários e institutos federais.

No cenário internacional, a liderança representa uma habilidade relacional imprescindível na enfermagem. Evidências na literatura têm mostrado que nas instituições em que liderança foi efetivamente ensinada houve um impacto positivo na prática. Tornam-se necessários maiores investimentos na formação da liderança de modo integrado ao currículo de Enfermagem, cabendo às instituições de ensino estimular o desenvolvimento de programas de liderança, além de buscar maior integração entre os serviços de saúde, com a finalidade de promover melhorias na prática e na cultura organizacional ${ }^{(3,21)}$.

Entretanto, na população estudada, o setor de atuação foi a única variável que obteve relação significativa com o estilo de liderança adotado. Observou-se que o estilo de liderança E2 (Persuadir) predominou nas UA, CME/CC e UI, dado também encontrado em outras pesquisas ${ }^{(1,6,9)}$.

No trabalho da enfermagem em unidades de cuidados, pesquisas ${ }^{(1,6,21)}$ apontam que a prática da liderança é influenciada positivamente pelo domínio da assistência, a excelência na prática clínica é considerada fator fundamental para a aceitação do enfermeiro como líder. Os achados estatísticos indicam que os enfermeiros no cenário investigado, apesar de próximos da assistência direta ao usuário, ao contrário do que menciona a literatura, têm adotado estilos que desfavorecem a liderança dialógica, afastando-se da equipe de trabalho. Apesar da jovialidade dos participantes e do pouco tempo de atuação, a liderança adotada pelos enfermeiros já sinaliza fragilidade, pois as tarefas ganham centralidade na prática profissional dos participantes. 
Esses dados indicam a necessidade de oferecer oportunidades aos enfermeiros líderes, estratégias de reconhecimento de seu perfil, tendo em vista que ao líder situacional são necessárias habilidades mínimas, entre elas a capacidade de diagnóstico, a flexibilidade e o saber trabalhar em equipe ${ }^{(7)}$. Ao se considerar o modelo de LS, não existe certo ou errado, pois esse consiste na premissa de que não existe um único estilo de liderança apropriado para toda e qualquer situação ${ }^{(1,7-8)}$. No entanto, cabe enfatizar que a atuação do enfermeiro, enquanto líder, precisa estar vinculada ao estabelecimento de relacionamentos interpessoais saudáveis, que priorizem, além das atividades, o bem-estar do trabalhador. Para tanto, o desenvolvimento da liderança não pode ser entendido como uma responsabilidade individual, visto que, para seu êxito, tornase imprescindível investimentos tanto das instituições de ensino quanto dos serviços de saúde.

O enfermeiro líder pode adotar diferentes condutas e ações no intuito de responsabilizar e envolver o grupo no processo de cuidar de modo articulado e coletivo, estabelecendo canais abertos de comunicação e desenvolvendo processos participativos de liderança, sensíveis às necessidades da equipe ${ }^{(16,21)}$. Contudo, o profissional precisa de condições para exercer sua liderança de modo a compreender o processo de trabalho que permeia seu cotidiano, reconhecendo peculiaridades de cada colaborador e da sua unidade, estabelecendo metas e procurando alcançá-las com a ajuda efetiva de sua equipe. Esse processo poderá ser facilitado com o auxílio da instituição, por meio da educação, bem como a partir da garantia de trabalhadores em quantidade adequada e de boas condições laborais.

\section{CONCLUSÕES}

Os resultados apontaram o predomínio do estilo Persuadir entre os enfermeiros no cenário hospitalar, o que ressalta a importância de discutir sobre a adoção de um estilo diretivo, no qual se evidencia o foco para a tarefa, o que pode limitar a criatividade e potencialidades dos integrantes da equipe. Apesar da população se destacar como jovem e com pouco tempo de atuação, a liderança adotada pelos enfermeiros já sinaliza fragilidade, sendo que as tarefas têm ganhado centralidade na sua prática.

O achado vai de encontro ao empoderamento dos envolvidos quanto a seu processo de trabalho, defendido na Teoria de LS. Isso pode interferir de modo negativo no processo de liderança no ambiente hospitalar, pois o predomínio da adoção dos estilos diretivos remete ao poder coercitivo, em que o líder não inspira seus colaboradores.

Contudo, a adoção de estilos LS relaciona-se diretamente com a maturidade dos membros da equipe, sua disposição em assumir a responsabilidade de dirigir seu próprio comportamento, e por isso compreendese como limitação do estudo a falta de dados sobre as percepções dos colaboradores, bem como informações relativas ao processo de trabalho dos enfermeiros, os quais poderiam fortalecer a avaliação especialmente quanto à adoção dos estilos diretivos e o próprio ambiente de trabalho, uma vez que podem influenciar o processo de liderar.

É fundamental a partir deste reconhecimento utilizar-se de estratégias que permitam alcançar elevados níveis de maturidade, oportunizando, desta forma, aos enfermeiros e colaboradores condições para 
que desenvolvam suas atividades com autonomia e que haja elevado nível de confiança entre os membros da equipe de enfermagem.

\section{REFERÊNCIAS}

1. Galvão CM, Trevizan MA, Sawada NO, Coleta JAD. Liderança situacional: estrutura de referência para o trabalho do enfermeiro-líder no contexto hospitalar. Rev. Latino-Am. Enfermagem. 1998;6(1):81-90.

2. Amestoy SC, Backes VMS, Trindade LL, Canever BP. The scientific production regarding leadership in the context of nursing. Rev. Esc. Enferm. USP. 2012;46(1):227-33.

3. Hanse JJ, Harlin U, Jarebrant C, Ulin K, Winkel J. The impact of servant leadership dimensions on leader-member exchange among health care professional. Journ Nurs Managem. 2016;24:228-34.

4. Trastek VF, Hamilton NW, Niles EE. Leadership models in health care - a case for servant leadership. Mayo Clinic Proceedings. 2014;89(3):374-81.

5. Paterson K, Henderson A, Burmeister E. The impact of a leadership development programme on nurses' selfperceived leadership capability. Journal of Nursing Management. 2015;23:1086-93.

6. González AL, Guevara SE, Morales FG, Segura HP, Luengo MC. Relación de la relación de la satisfacción laboral con estilos de liderazgo en enfermeros de hospitales públicos, Santiago, Chile. Cienc. enferm. 2013;19(1):11-21.

7. Hersey $\mathrm{P}$, Blanchard K. Psicologia para administradores: a teoria e às técnicas da liderança situacional. São Paulo: EPU; 1986.

8. Blanchard K. Liderança de alto nível: como criar e liderar organizações de alto desempenho. Porto Alegre: Bookman; 2011.

9. Torres-Contreras CC. Liderazgo situacional en enfermeras de una institución de salud de Bucaramanga. Enfermería Clínica. 2013;23(4):140-7.

10. Veliz RL, Paravic KT. Coaching educativo como estrategia para fortalecer El liderazgo en enfermería. Cienc. enferm. 2012;18(2):111-7.

11. Hudgins TA. Resilience, job satisfaction and anticipated turnover in nurse leaders. Journ Nurs Management. 2016;24:E62-9.

12. Hersey P, Blanchard KH. LEAD Self. Escondido, CA: Center for Leadership Studies; 1973.

13. Galvão CM. Liderança situacional: uma contribuição ao trabalho do enfermeiro-líder no contexto hospitalar [tese]. São Paulo: Universidade Federal de São Paulo, Escola de Enfermagem de Ribeirão Preto; 1995.

14. Griep RH, Fonseca MDJMD, Melo ECP, Portela LF, Rotenberg L. Enfermeiros dos grandes hospitais públicos no Rio de Janeiro: características sociodemográficas e relacionadas ao trabalho. Rev. bras. Enferm. 2013;66:151-7.

15. Conselho Federal de Enfermagem. Total de profissionais de enfermagem no Brasil. [Internet]. 2015. Disponível em: http://www.cofen.gov.br/oficina-discute-politicas-publicas-a-luz-da-pesquisa-perfil-da-

enfermagem_33599.html.com.br/estatisticas.asp.

16. Regis LF, Porto IS. Basic human needs of nursing professional: situations of (dis)satisfaction at work. Rev EsC Enferm USP. 2011;45(2):332-8.

17. Moura GMSS, Inchauspe JAF, Dall'Agnol CM, Magalhães AMM, Hoffmeister LV. Expectativas da equipe de enfermagem em relação à liderança. Acta paul enferm. 2013;26(2):198-204.

18. Yañez-Gallardo R, Valenzuela-Suazo S. Critical incidents of trust erosion in leadership of head nurses. Rev. LatinoAm. Enfermagem. 2012;20(1):143-50.

19. Fleiszer AR, Semenic SE, Ritchie JA, Richer MC, Denis JL. Nursing unit leaders' influence on the long-term sustainability of evidence-based practice imporvement. Journal Nursing Management. 2016;24:309-18.

20. Dignam D, Duffield C, Stasa H, Gray J, Jackson D, Daly J. Management and leadership in nursing: an Australian educational perspective. Journal of Nursing Management. 2012;20:65-71.

21. Curtis EA, Sheerin FKS, Vries, JAN. Developing leadership in nursing: the impact of education and training. British Journal of Nursing. 2011;20(6):344-52. 